\title{
INFLUENCE OF CONSERVATION TILLAGE AND POST-EMERGENCE HERBICIDES ON WEED CONTROL AND COTTON YIELD UNDER WHEAT-COTTON SYSTEM
}

Niamat Ullah Khan ${ }^{1 *}$, Azhar Abbas Khan², Zeshan Hassan², Umbreen Shahzad ${ }^{2}$, Muhammad Arshad Farooq ${ }^{3}$, Malik Fahim Abbas $^{4}$, Aamir Roheen ${ }^{4}$

\begin{abstract}
Field experiments were conducted to evaluate the performance of cotton under three tillage systems, viz., ZT, RT and CT; and five herbicides, i.e., haloxyfop-R-methyl $10.8 \mathrm{EC}$ (108 g a.i. ha ${ }^{-1}$ ), lactofen 24 EC (168 g a.i. ha-1), haloxyfop $10.8 \mathrm{EC}+$ lactofen $24 \mathrm{EC}$, hand weeding, and weedy check during 2017 and 2018 at Cotton Research Station, D.I.Khan, Pakistan, and to explore the best management option for effective weed control and enhanced yield of cotton grown after wheat. The results revealed that hand weeding and Floxyfop as post emergence alone or in combination with Lactofen reduced weed density and weed biomass to the minimum irrespective of the tillage systems. Haloxyfop + lactofen produced higher seed cotton yield in RT than ZT and CT. Maximum weed population reduction percentage was recorded under haloxyfop+ lactofen. Broad-spectrum herbicides $x$ RT produced the highest seed cotton yield. Weed density and weed biomass were also lowest in broad-spectrum herbicides $\times$ RT. In conclusion, broad-spectrum herbicides under RT were more effective in wheat based cropping system of D.I.Khan. It is concluded from present data that reduced tillage is more productive than zero tillage and conventional tillage and conservation tillage may improve yield of cotton in addition to improvement in soil quality if severe weeds are not infesting the crop.
\end{abstract}

Key words: cotton, tillage, herbicides, yield, weed density, weed biomass

Citation: Khan, N.U.; A.A. Khan; Z. Hassan; U.Shahzad; M. A. Farooq; M. F. Abbas; A. Roheen. 2021. Influence Of Conservation Tillage And Post-Emergence Herbicides On Weed Control And Cotton Yield Under Wheat-Cotton System. Pak. J. Weed Sci. Res., 27 (3):397406

$1^{*}$ PCCC, Cotton Research Station, Dera Ismail Khan

${ }^{2}$ College of Agriculture, Bahaudin Zakria University, Bahadur Campus, Layyah.

${ }^{3}$ PARC, Pakistan Agricultural Research Council, Islamabad.

${ }^{4}$ Department of Agronomy, Faculty of Agriculture, Gomal University, Dera Ismail Khan

*Corresponding author. Email: drkhancrs@gmail.com 


\section{Introduction}

Conservation tillage is one such good management practice that leaves $30 \%$ or more of the crop residues on the soil surface after planting, which may enhance soil fertility, water and nutrient use efficiency, and reduce production cost, and may enhance yield and quality of cotton in the long run (CTIC 1998; Hulugalle et al. 2004). Unlike CT, conservation tillage is considered to be a safe practice regarding its impact on soil, water, and environment (Edwards \& Daniel 1992). Conservation tillage can alleviate soil-related constraints, while CT may deteriorate soil structure, accelerate erosion, and deplete soil organic matter (SOM) and fertility of the soil (Lal 1993).Conservation tillage is a suitable tillage method that increased soil fertility, decreased weed density and cotton production as compared to conventional tillage (Endale et al., 2002). $C_{S}$ T impacted soil, water and environment. Conservation tillage plus wheat-straw economically increased fertility and yield (Blaise and Ravindran. 2003). Conservation tillage is the possible alternative to builds up organic matter in the surface of soil, increase moisture absorption capability, improve soil prosperities and increased cotton yield as compared RT to conventional tillage (Buman et al., 2005). RT plus residues enhanced SOM and potassium in the soil (Bauer et al., 2010). Cotton scientists said that RT with residue mulch gave higher size of aggregates, stability, total organic carbon in soil aggregates and lower weed density than conventional tillage (Balkcom et al., 2010). Higher accumulation of organic matter in the soil, greater fertilizer use efficiency and decreased weed biomass increased cotton yield under conservation tillage (Aulakh et al., 2011). Crop residues on soil surface in conservation tillage recommended for moisture conservation in cotton sown after wheat (Balkcom et al., 2006). Conservation tillage with straw mulch enhanced fertilizer agronomic efficiency and crop yield (Blaise, 2006; Atreya et al., 2006). Conservation tillage increased microbial biomass and produced higher weed population reduction percentage (Mohler et al., 2006).

Weeds are another problem that significantly affected cotton yields (Ali et al., 2013). Cotton crop requires optimum weeding for maximum production. Post emergence herbicides greatly influenced weed density, and biomass resulted in higher cotton yield (Cardina et al., 2007).

Higher weed density showed fewer bolls per plant and boll weight in conservation tillage cotton (Balkcom et al., 2010; Chinnusamy et al., 2013). Residues mulch in conservation tillage could reduce weed density and improved nutrients availability (Dadari and Kuchinda, 2004; Culpepperet al., 2009;Aulakh et al. 2011). Mostly manual weeding is done in conventional tillage which is costly and laborious (Buman et al., 2005; Deshpandeet al. 2006). Weed problem is solved in conservation tillage as with the introduction of post emergence herbicides such as floxyfop (Grichar et al., 2004; Hallikeriet al., 2004; Blaise, 2006; Atreya et al., 2006; Holloway et al., 2008). Post emergence herbicides use in conventional tillage cotton has proved un-productive (Paulsgrove et al., 2005; Balkcom et al., 2010; Prabhuet al., 2011). Research findings showed that the conservation tillage with post emergence herbicides would be a possible alternative for sustainable cotton crop production in irrigated wheat based cotton system (Mohler et al., 2006; Shaikhet al., 2006; Riar, et al., 2011). The current experiment was carried out with the aim to investigate the impact of tillage, postemergence herbicides and their interaction on weed control and seed cotton yield in wheat based cotton system of Dera Ismail Khan.

\section{Materials and methods}

\section{Experimental site}

Field trials were carried out during 2017 and 2018 at Cotton Research Station, Pakistan Central cotton Committee, Dera Ismail Khan, Pakistan. The area is hot and dry, main features of the area. Weather data is shown in Table 1. 


\section{Experimental procedure}

Field trials were conducted to evaluate the impact of tillage and post emergence weed control treatments or herbicides on weed density, yield and quality of cotton in wheat based cropping system for two consecutive years in 2017 and 2018. Wheat was the previous crop at the experimental site in both years. Wheat (cv. Hashim-8, a standard variety of the region) was sown in the 2nd week of December under irrigated conditions during both years of study. Wheat crop was fertilized with $120 \mathrm{~kg} \mathrm{~N}, 60 \mathrm{~kg} \mathrm{P}$ and $30 \mathrm{~kg} \mathrm{~K} \mathrm{ha}{ }^{-1}$. After wheat harvest, field was irrigated. When field came in proper moisture condition, previous crop residues were incorporated into the soil with ploughing operations including tiller, disc plough and rotavator and after well prepared seed bed, cotton was sown with dibbling method (conventional tillage system, CT). The reduced tillage (RT) system consisted of a tiller, while in the zero tillage plots; cotton was directseeded into wheat residue. Cotton (cv. Ali Akbar, a standard Bt variety of cotton for the region) was sown at $75 \mathrm{~cm}$ inter-row and $22.5 \mathrm{~cm}$ intra row spacing with dibbling method on May 7, 2017 and May 8, 2018, respectively. Three tillage systems viz. zero tillage ( $\mathrm{ZT})$, reduced tillage (RT), conventional tillage (CT) and five post emergence weed control treatments or herbicides, i.e., floxyfop
10.8 EC (haloxyfop -R-methyl @ $108 \mathrm{~g}$ a.i. $\left.\mathrm{ha}^{-1}\right)$, lactofen 24 EC (168 g a.i. ha ${ }^{-1}$ ), floxyfop (108 g a.i. ha ${ }^{-1}$ ) + lactofen 24 EC (168 g a.i. ha ${ }^{-1}$ ), hand weeding, and weedy check (untreated control) were applied in randomized complete block design with split plot combined over years and four replications. Hand weeding was done $30 \mathrm{~d}$ after sowing once in a season each year. Tillage was allotted to main plots, while herbicides were applied to subplots. The size of each subplot was 10 $\mathrm{m} \times 3 \mathrm{~m}$ having four rows and $10 \mathrm{~m}$ long. A standard dose of 150:90 kg N:P ha ${ }^{-1}$ was used in the form of urea and triple supper phosphate. All the phosphorous and half of the $\mathrm{N}$ were applied with sowing, while remaining half of the $\mathrm{N}$ was applied in two splits at the 1st and 2nd irrigation. Six irrigations were applied at about 15 day interval from the beginning of square stage to the bolls during the growing season each year. The crop was harvested on December 4, 2017 and December 5, 2018 during 2 years of experimentation.

\section{Statistical analysis}

Statistical analyses of the data were performed as per ANOVA techniques (Steel et al., 1980) and significant results were subjected to LSD test for mean comparison using MSTATC software (MSTATC, 1991).

Table 1 Meteorological data recorded at Cotton Research Station, Dera Ismail Khan during 2017 and 2018

\begin{tabular}{|c|c|c|c|c|c|c|c|c|}
\hline \multicolumn{5}{|c|}{2017} & \multicolumn{4}{|c|}{2018} \\
\hline \multirow{2}{*}{ Month } & \multicolumn{3}{|c|}{ Temp. ${ }^{\circ} \mathrm{C}$} & \multirow{2}{*}{ Rainfall (mm) } & \multicolumn{3}{|c|}{ Temp. ${ }^{\circ} \mathrm{C}$} & \multirow{2}{*}{ Rainfall (mm) } \\
\hline & Max & Min & Average & & Max & Min & Average & \\
\hline April & 39 & 20 & 29.5 & 13.5 & 45 & 17 & 37.5 & - \\
\hline May & 41 & 25 & 33 & 22 & 43 & 25 & 38 & - \\
\hline June & 42 & 27 & 34.5 & 10 & 44 & 27 & 39.25 & 35 \\
\hline July & 37 & 27 & 32 & 35 & 39 & 27 & 35.5 & 124 \\
\hline
\end{tabular}




\begin{tabular}{|c|c|c|c|c|c|c|c|c|}
\hline August & 36 & 26 & 31 & 52 & 38 & 29 & 34.75 & 173 \\
\hline September & 37 & 24 & 30.5 & 19 & 37 & 25 & 33.5 & 20 \\
\hline October & 35 & 20 & 27.5 & 5 & 34 & 19 & 29.5 & - \\
\hline November & 28 & 10 & 19 & - & 29 & 14 & 24.5 & - \\
\hline \multicolumn{4}{|c|}{ Total rainfall } & 156.5 & & & & 352 \\
\hline
\end{tabular}

Source: Arid Zone Research Institute, Dera Ismail Khan, Pakistan.

Table 2. Mean square values of weed density, dry weeds biomass, weed population reduction (\%)and seed cotton yield as affected by tillage and herbicides application

\begin{tabular}{|c|c|c|c|c|c|c|}
\hline Year & Source & D.F. & $\begin{array}{l}\text { Weed } \\
\text { density } \mathrm{m}^{-2}\end{array}$ & $\begin{array}{l}\text { Dry weed } \\
\text { biomass } \mathrm{g} \mathrm{m}^{-2}\end{array}$ & $\begin{array}{l}\text { Weed } \\
\text { reduction } \\
(\%)\end{array}$ & $\begin{array}{l}\text { seed cotton yield } \\
\text { kgha }^{-1}\end{array}$ \\
\hline \multirow{6}{*}{2017} & Rep.. & 3 & 0.163 & 3.162 & 1.910 & 5668.728 \\
\hline & Tillage-T & 2 & 616.683 & 1940.209 & 521.063 & 763405.067 \\
\hline & Error (a) & 6 & 0.232 & 6.384 & 0.535 & 26888.711 \\
\hline & $\begin{array}{l}\text { Herbicides- } \\
\mathrm{H}\end{array}$ & 4 & 9998.632 & 61485.472 & 9737.076 & 3696361.233 \\
\hline & $\mathrm{T} \times \mathrm{H}$ & 8 & 285.920 & 270.890 & 62.701 & 216858.608 \\
\hline & Error (b) & 36 & 0.121 & 5.734 & 1.493 & 13052.106 \\
\hline \multirow{6}{*}{2018} & Rep.. & 3 & 0.026 & 5.249 & 1.521 & 13301.644 \\
\hline & Tillage-T & 2 & 758.540 & 1093.866 & 457.333 & 714473.750 \\
\hline & Error (a) & 6 & 0.111 & 9.102 & 3.667 & 7031.728 \\
\hline & $\begin{array}{l}\text { Herbicides- } \\
\mathrm{H}\end{array}$ & 4 & 9638.946 & 47199.221 & 9293.854 & 7386243.900 \\
\hline & $\mathrm{T} \times \mathrm{H}$ & 8 & 310.936 & 267.519 & 70.500 & 61909.688 \\
\hline & Error (b) & 36 & 0.247 & 4.877 & 1.748 & 16037.547 \\
\hline
\end{tabular}

\section{Results}

\section{Weed density $\mathbf{m}^{-2}$ after application of} herbicides

ANOVA indicated that weed density was significantly by tillage $(T)$, herbicides $(H)$ and tillage and herbicide interaction (Table-2). Reduced tillage had the lowest

\section{Weed population reduction (WPR \%)}

WPR \% showed significant response to $T$, $\mathrm{H}$ and $\mathrm{T} \times \mathrm{H}$ interactive effects during 2017 and 2018 (Table 2). Means showed that weed density as compared to zero and conventional tillage. Mean data for herbicide revealed that floxyfop + lactofen gave lowest weed density amongst all the other herbicides treatments (Table 3). Interactions indicated that reduced tillage with floxyfop + lactofen produced minimum weed density.

reduced tillage had the highest WPR \% (Table 4). Floxyfop + lactofen resulted in more WPR\% (Table 4). Reduced tillage with broad-spectrum herbicide gave maximum WPR \% than others. 
Table 3 Weeds density $\left(\mathrm{m}^{-2}\right)$ as affected by tillage and herbicide during 2017 and 2018

\begin{tabular}{|c|c|c|c|c|c|}
\hline \multirow[t]{3}{*}{ Year } & \multirow[t]{3}{*}{ Herbicides } & \multicolumn{3}{|c|}{ Tillage } & \multirow[t]{3}{*}{ Means } \\
\hline & & \multicolumn{2}{|c|}{ Conservation } & Conventional & \\
\hline & & Zero & Reduced & & \\
\hline \multirow[t]{7}{*}{2017} & Haloxyfop & $6.80 \mathrm{hij}$ & $8.47 \mathrm{~g}$ & $7.40 \mathrm{ghi}$ & $7.56 \mathrm{c}$ \\
\hline & Lactofen & $40.40 \mathrm{e}$ & $33.60 \mathrm{f}$ & $56.50 \mathrm{c}$ & $43.50 \mathrm{~b}$ \\
\hline & Haloxyfop + lactofen & $3.50 \mathrm{k}$ & $3.43 \mathrm{k}$ & $3.33 \mathrm{k}$ & $3.42 \mathrm{~d}$ \\
\hline & Manual weeding & $5.60 \mathrm{j}$ & $8.33 \mathrm{gh}$ & $6.47 \mathrm{ij}$ & $6.80 \mathrm{c}$ \\
\hline & Control & $64.57 \mathrm{~b}$ & $53.43 \mathrm{~d}$ & $87.43 \mathrm{a}$ & $68.48 a$ \\
\hline & Tillage means & $24.17 \mathrm{~b}$ & $21.45 \mathrm{c}$ & $32.23 \mathrm{a}$ & \\
\hline & \multicolumn{5}{|c|}{ LSD0.05 for tillage $=0.6121$, herbicides $=0.9682$, tillage $\times$ herbicides $=1.6770$} \\
\hline \multirow[t]{7}{*}{2018} & Haloxyfop & $4.57 \mathrm{ij}$ & $7.53 \mathrm{~g}$ & $5.47 \mathrm{hij}$ & $5.86 \mathrm{c}$ \\
\hline & Lactofen & $39.50 \mathrm{e}$ & $29.53 \mathrm{f}$ & $54.57 \mathrm{c}$ & $41.20 \mathrm{~b}$ \\
\hline & Haloxyfop + lactofen & $5.63 \mathrm{hij}$ & $3.80 \mathrm{j}$ & $4.47 \mathrm{ij}$ & $4.63 d$ \\
\hline & Manual weeding & $5.13 \mathrm{hij}$ & $6.23 \mathrm{ghi}$ & $6.63 \mathrm{gh}$ & $6.00 \mathrm{c}$ \\
\hline & Control & $64.40 \mathrm{~b}$ & $51.20 \mathrm{~d}$ & $87.37 \mathrm{a}$ & $67.66 \mathrm{a}$ \\
\hline & Tillage means & $23.85 \mathrm{~b}$ & $19.66 \mathrm{c}$ & $31.70 \mathrm{a}$ & \\
\hline & \multicolumn{5}{|c|}{ LSD0.05 for tillage $=0.9249$, herbicides $=1.0773$, tillage $\times$ herbicides $=1.8659$} \\
\hline
\end{tabular}
Averages having similar letters are not significant at 5 percent level of probability

Table 4Weed population reduction (\%) as affected by tillage and herbicide during 2017 and 2018

\begin{tabular}{|c|c|c|c|c|c|}
\hline \multirow[t]{3}{*}{ Year } & \multirow[t]{3}{*}{ Herbicides } & \multicolumn{3}{|c|}{ Tillage } & \multirow[t]{3}{*}{ Means } \\
\hline & & \multicolumn{2}{|c|}{ Conservation } & Conventional & \\
\hline & & Zero & Reduce & & \\
\hline \multirow[t]{7}{*}{2017} & Haloxyfop & $65.42 \mathrm{~h}$ & $67.20 \mathrm{~g}$ & $48.24 \mathrm{i}$ & $60.29 c$ \\
\hline & Lactofen & $29.35 \mathrm{k}$ & $31.44 \hat{j}$ & 18.33 I & $26.37 \mathrm{~d}$ \\
\hline & Haloxyfop + lactofen & $92.41 \mathrm{~b}$ & $96.19 \mathrm{a}$ & $90.51 \mathrm{c}$ & $93.04 \mathrm{a}$ \\
\hline & Manual weeding & $77.19 \mathrm{e}$ & $79.18 \mathrm{~d}$ & $74.19 \mathrm{f}$ & $76.85 b$ \\
\hline & Control & - & - & - & - \\
\hline & Tillage means & $52.87 b$ & $54.80 \mathrm{a}$ & $46.25 \mathrm{c}$ & \\
\hline & \multicolumn{5}{|c|}{ LSD0.05 for tillage $=0.8619$, herbicides $=0.9610$, tillage $\times$ herbicides $=1.6644$} \\
\hline \multirow[t]{7}{*}{2018} & Haloxyfop & $70.31 \mathrm{~h}$ & $74.20 \mathrm{~g}$ & $52.44 \mathrm{i}$ & $65.65 \mathrm{c}$ \\
\hline & Lactofen & $30.35 \mathrm{k}$ & $34.31 \mathrm{j}$ & 25.511 & $30.06 \mathrm{~d}$ \\
\hline & Haloxyfop + lactofen & $94.40 \mathrm{~b}$ & $97.44 \mathrm{a}$ & $91.40 \mathrm{c}$ & $94.41 \mathrm{a}$ \\
\hline & Manual weeding & $81.16 \mathrm{e}$ & $84.23 \mathrm{~d}$ & $78.54 \mathrm{f}$ & $81.31 \mathrm{~b}$ \\
\hline & Control & - & - & - & - \\
\hline & Tillage means & $55.24 \mathrm{~b}$ & $58.04 \mathrm{a}$ & $49.58 \mathrm{c}$ & \\
\hline & SD0.05 for ti & 1 herhis & $=0736$ & $200 \times$ ho & 59 \\
\hline
\end{tabular}

Averages having similar letters are not significant at 5 percent level of probability

\section{Dry weed biomass $\left(\mathrm{g} \mathrm{m}^{-2}\right)$}

Dry weed biomass (DWB) was influenced significantly by $\mathrm{T}, \mathrm{H}$, and $\mathrm{T} \times \mathrm{H}$ interaction in 2017 and 2018 (Table- 3). Reduced tillage produced lowest DWB compared conventional tillage (Table 6). Minimum
DWB was recorded with floxyfop + lactofen as compared to all other combinations. Interactions revealed that reduced tillage with broad spectrum herbicide gave maximum DWB. 
Table 5Dry weed biomass ( $\mathrm{g} \mathrm{m}^{-2}$ )as affected by tillage and herbicide during 2017 and 2018

\begin{tabular}{|c|c|c|c|c|c|}
\hline \multirow[t]{3}{*}{ Year } & \multirow[t]{3}{*}{ Herbicides } & \multicolumn{3}{|c|}{ Tillage } & \multirow[t]{3}{*}{ Means } \\
\hline & & \multicolumn{2}{|c|}{ Conservation } & Conventional & \\
\hline & & Zero & Reducec & & \\
\hline \multirow{7}{*}{2017} & Haloxyfop & $29.40 \mathrm{i}$ & $26.27 \mathrm{j}$ & $34.63 \mathrm{~g}$ & $30.10 \mathrm{c}$ \\
\hline & Lactofen & $124.50 \mathrm{e}$ & $97.57 \mathrm{f}$ & $132.37 \mathrm{~d}$ & $118.14 b$ \\
\hline & Haloxyfop + lactofen & $8.17 \mathrm{~m}$ & $5.40 \mathrm{n}$ & $10.50 \mid$ & $8.02 \mathrm{e}$ \\
\hline & Manual weeding & $29.17 \mathrm{i}$ & $22.47 \mathrm{k}$ & $32.37 \mathrm{~h}$ & $28.00 \mathrm{~d}$ \\
\hline & Control & $178.64 \mathrm{~b}$ & $153.67 \mathrm{c}$ & $191.50 \mathrm{a}$ & $174.60 \mathrm{a}$ \\
\hline & Tillage means & $73.98 \mathrm{~b}$ & $61.07 \mathrm{c}$ & $80.27 \mathrm{a}$ & \\
\hline & \multicolumn{5}{|c|}{ LSD0.05 for tillage $=0.7210$, herbicides $=0.9914$, tillage $\times$ herbicides $=1.7172$} \\
\hline \multirow[t]{7}{*}{2018} & Haloxyfop & $26.30 \mathrm{~g}$ & $20.47 \mathrm{i}$ & $26.43 \mathrm{~g}$ & $24.40 \mathrm{c}$ \\
\hline & Lactofen & $97.50 \mathrm{e}$ & $80.73 \mathrm{f}$ & $117.60 \mathrm{~d}$ & $98.61 \mathrm{~b}$ \\
\hline & Haloxyfop + lactofen & $5.73 \mathrm{j}$ & $4.47 \mathrm{j}$ & $5.63 \mathrm{j}$ & $5.28 \mathrm{~d}$ \\
\hline & Manual weeding & $23.30 \mathrm{~h}$ & $21.60 \mathrm{hi}$ & $25.37 \mathrm{~g}$ & $23.42 \mathrm{c}$ \\
\hline & Control & $157.80 \mathrm{~b}$ & $136.73 \mathrm{c}$ & $164.03 \mathrm{a}$ & $152.86 \mathrm{a}$ \\
\hline & Tillage means & $62.13 \mathrm{~b}$ & $52.80 \mathrm{c}$ & $67.81 \mathrm{a}$ & \\
\hline & \multicolumn{5}{|c|}{ LSD0.05 for tillage $=0.6923$, herbicides $=1.1707$, tillage $\times$ herbicides $=2.0277$} \\
\hline
\end{tabular}
Averages having similar letters are not significant at 5 percent level of probability

Seed cotton yield $\mathrm{kgha}^{-1}$

Seed cotton yield had significant response to $T, H$, and $T \times H$ interactions during 2017 and 2108 (Table-2). Reduced tillage had significantly greater yield than Zero and conventional tillage in 2017 and 2018 (Table 7). Herbicide means revealed Table 6 Seed cotton yield $\left(\mathrm{kg} \mathrm{ha}^{-1}\right)$ as affected by tillage and herbicide during 2017 and 2018

\begin{tabular}{|c|c|c|c|c|c|}
\hline \multirow[t]{3}{*}{ Year } & \multirow[t]{3}{*}{ Herbicides } & \multicolumn{3}{|c|}{ Tillage } & \multirow[t]{3}{*}{ Means } \\
\hline & & \multicolumn{2}{|c|}{ Conservation } & Conventional & \\
\hline & & Zero & Reduced & & \\
\hline \multirow[t]{7}{*}{2017} & Haloxyfop & $2560.3 d$ & $2648.0 d$ & $2226.3 e$ & $2478.2 c$ \\
\hline & Lactofen & $2376.3 \mathrm{e}$ & $2659.3 d$ & $2535.0 d$ & $2523.6 \mathrm{c}$ \\
\hline & Haloxyfop + lactofen & $3227.0 a b$ & $3312.3 a$ & $3138.0 b$ & $3225.8 \mathrm{a}$ \\
\hline & Manual weeding & $2862.3 c$ & $3137.3 a b$ & $2958.3 c$ & $2986.0 \mathrm{~b}$ \\
\hline & Control & 1963.0f & $2231.0 \mathrm{e}$ & $1160.3 \mathrm{~g}$ & $1784.8 d$ \\
\hline & Tillage means & $2597.8 b$ & $2797.6 \mathrm{a}$ & $2403.6 \mathrm{c}$ & \\
\hline & \multicolumn{5}{|c|}{ LSD0.05 for tillage $=15.220$, herbicides $=108.09$, tillage $\times$ herbicides $=168.10$} \\
\hline \multirow[t]{7}{*}{2018} & Haloxyfop & $3065.0 \mathrm{e}$ & $3333.0 \mathrm{~d}$ & $2762.0 \mathrm{f}$ & $3053.3 c$ \\
\hline & Lactofen & $2758.0 \mathrm{f}$ & 2956.7 e & $2658.0 \mathrm{f}$ & $2790.9 \mathrm{~d}$ \\
\hline & Haloxyfop + lactofen & $4042.7 \mathrm{~b}$ & $4224.3 \mathrm{a}$ & $3522.0 \mathrm{c}$ & $3929.7 \mathrm{a}$ \\
\hline & Manual weeding & $3441.0 \mathrm{~cd}$ & $3521.3 \mathrm{c}$ & $3311.0 \mathrm{~d}$ & $3424.4 b$ \\
\hline & Control & $1834.7 \mathrm{gh}$ & $1949.3 \mathrm{~g}$ & $1739.3 \mathrm{~h}$ & $1841.1 \mathrm{e}$ \\
\hline & Tillage means & $3028.3 \mathrm{~b}$ & $3196.9 \mathrm{a}$ & $2798.5 \mathrm{c}$ & \\
\hline & \multicolumn{5}{|c|}{ LSD0.05 for tillage $=43.182$, herbicides $=103.63$, tillage $\times$ herbicides $=179.50$} \\
\hline
\end{tabular}

Averages having similar letters are not significant at 5 percent level of probability 


\section{Discussion}

Conservation tillage in cotton after wheat is on experimental basis in Pakistan. Though, cotton growers take eager attention in conservation tillage cotton establishment after wheat harvest due to lower cost of production and profitable cotton yield (Buman et al., 2005). Research data revealed that lower weed density observed in conservation tillage (CST) and greater population reduction percentage resulted in maximum seed cotton yield than conventional tillage. Enhanced seed cotton in conservation tillage could be due to increased weeds reduction percentage probably due to completely undisturbed soil and improved soil fertilizer and soil organic matter (Mohler et al., 2006). Greater weed population reduction (WPR \%) under conservation tillage contributed to yield improvements compared to the conventional tillage (Aulakh et al. 2011). In long-term study on conservation tillage, significant yield differences were observed in upland cotton (Balkcom et al., 2010).The study offers great yield variations in cotton genotype under conservation tillage. In addition, improved soil moisture content due straw mulch and better soil physical conditions might have contributed to more yield improvements in conservation tillage than conventional tillage (Bauer et al., 2010). Buman et al. (2005) obtained greater yield due to better soil hydrothermal regime under conservation tillage and post herbicides. In this research work, seed cotton yields was significantly encouraged by conservation tillage plots compared to conventional plots. Seed cotton yield of the conservation tillage with residue plots was significantly higher compared with the conventional tillage treatments due to the distinctive decreased dry weed biomass (Balkcom et al., 2010; Chinnusamy et al., 2013). Our results revealed greater seed cotton-yields in residues retained plots maybe due to improved nutrient accessibility in crop residues and right fertilizer management in soil through the adjustment of abundant micro-organisms after returning of wheat residues to the cotton fields (Dadari and Kuchinda, 2004; Culpepper et al., 2009; Aulakh et al. 2011). Scientific results showed that broad spectrum herbicides produced maximum seed cotton yield (Buman et al., 2005; Deshpandeet al., 2006). Enhanced yields in conservation tillage with floxyfop + lactofenwere probably due to decreased weed density, improved decomposition of wheat straw that enhances biological activity, better cotton root growth because of improved soil structure and enhanced soil moisture content by way of better infiltration rates (Grichar et al., 2004; Hallikeriet al., 2004; Blaise, 2006; Atreya et al., 2006; Holloway et al., 2008).

\section{Conclusion}

Application of floxyfop + lactofen as post emergence and hand weeding effectively controlled both narrow- and broad-leaved weeds regardless of the tillage system. However, floxyfop + lactofen in combination with RT were more effective and economical than $\mathrm{ZT}$ and $\mathrm{CT}$. Thus it can be concluded that post emergence broads spectrum herbicides (floxyfop + lactofen) in combination with RT is more appropriate strategy to control weed and enhance cotton yield in WBCS of Dera Ismail Khan, Pakistan. 


\section{References}

Atreya, K., S. Sharma, R.M. Bajracharya and N.P. Rajbhandari. 2006. Applications of Reduced Tillage in Hills of Central Nepal. Soil Till. Res. 88(1-2): 16-29.

Ali, H., S. A. Abid, S. Ahmad, N. Sarwar, M. Arooj, A. Mahmood and A.N. Shahzad. 2013. Impact of integrated weed management on flat-sown cotton (Gossypiumhirsutuml.). J. Anim. Plant Sci. 23(4): 1185-1192.

Aulakh, J.S., A.J. Price, K.S. Balkcom. 2011. Weed Management and Cotton Yield under Two Row Spacings in Conventional and Conservation Tillage Systems Utilizing Conventional, Glufosinate-, and Glyphosate-based Weed Management Systems. Weed Tech. 25(4): 542-547.

Balkcom, K.S., D.W. Reeves., J.N. Shaw, C. H. Burmester, L.M. Curtis. 2006. Cotton yield and fiber quality from irrigated tillage systems in the Tennessee Valley. Agron. J. 98(3): 596-602.

Balkcom, S. Kipling, A.J. Price, V. Santen, E. Delaney, P. Dennis, Boykin, L. Deborah, Arriaga, J. Francisco, Bergtold, S. Jason, Kornecki, S. Ted, Raper, L. Randy. 2010. Row spacing, tillage system, and herbicide technology affects cotton plant growth and yield. 117 (2-3): 219225.

Bauer, J.P. Fortnum, A. Bruce, R. James. 2010. Cotton responses to tillage and rotation during the turn of the century drought. Agron. J. 102(4): 1145-1148.

Blaise, D., C.D. Ravindran. 2003. Influence of tillage and residue management on growth and yield of cotton grown on a Vertisol over 5 years in a semi-arid region of India. Soil Till. Res. 70, 163-173.

Blaise, D. 2006. Effect of tillage systems on weed control, yield and fibre quality of upland (Gossypiumhirsutum L.) and Asiatic tree cotton (G. arboreum L.) Soil Till.Res. 91: 207-216.

Buman, R.A., B.A. Alesii, J.F. Bradley, J.L. Hatfield, D.L. Karlen. 2005. Profit and yield of tillage in cotton production systems. J. Soil. Water Cons. 60(5): 235-242.

Cardina, J., C.P. Herms, D.J. Doohan. 2002. Crop rotation and tillage system effects on weed seedbanks. Weed Sci 50: 448460.

Chinnusamy, N., C. Chinnagounder, P.N. Krishnan. 2013. Evaluation of weed control efficacy and seed cotton yield in glyphosate tolerant transgenic cotton. American J. Plant Sci. 4 (6): 1159-1163.

Culpepper, A.S., and A.C. York. 2000. Weed management in ultra narrow row cotton (Gossypiumhirsutum). Weed Technol. 14:19-29.

Dadari S. A. and N.C. Kuchinda. (2004) Evaluation of some pre- and postemergence weed control measures on rain-fed cotton (Gossypiumhirsutum L) in Nigerian savannah. Crop Protection, 23: 457461.

Deshpande, R.M., W.S. Pawar, P.S. Mankar, P.N. Bolde, A.N. Chinote. 2006. In tegrated weed management in rainfed cotton (GossypiumhirsutumL.). Ind. J. Agron. 15(1): 68-69.

Endale, D.M., M.L. Cabrera, J.L. Steiner, D.E. Radcliffe, W.K. Vencill, H.H. Schomberg, L. Lohr. 2002. Impact of conservation tillage and nutrient management on soil water and yield of cotton fertilized with poultry litter or ammonium nitrate in the Georgia Piedmont. Soil Till.Res. 66:55- 68.

Grichar, W.M., B.A. Besler, D.B. Kevin, B.W. Minton. 2004. Using soilapplied herbicides in combination with glyphosate in a glyphosate- 
resistant cotton herbicide program. Crop Protection 23:1007-1010.

Hallikeri, S.S., H.L. Halemani, and R.A. Nandagavi. 2004. Integrated Weed Management in Cotton Under Assured Rainfall Situation.Karnataka J. Agric. Sci.17 (4):663-669.

Holloway, J., L. Trolinder, J.M. Ellis and S. Baker. 2008. New herbicide tolerance technology for glyphosate resistant weed management in cotton. Proc. Beltwide Cotton Prod. Res. Conf., Nashville, TN. 8-11 Jan. 2008. Natl.Cotton Counc. Am., Memphis, TN. pp-1726.

Mohler, C.L., J.C. Frisch, C.E. McCulloch. 2006. Vertical movement of weed seed surrogates by tillage implements and natural processes. Soil Till. Res. 86, 110-122.

Norsworthy, J. K. 2008. Effect of tillage intensity and herbicide programs on changes in weed species density and composition in the southeastern coastal plains of the United States.Crop Protection.27: 151-160.

Norsworthy, K., J. McClelland, M. Griffith, Bangarwa, K. Sanjeev, S. Joshua. 2010. Evaluation of legume cover crops and weed control programs in conservation-tillage enhanced glyphosate-resistant cotton. Weed Tech. 24(3): 269-274.

Papageorgiou, I. Eleftherohorinos, I. Vasilakoglou, Ioannis. 2008. Tillage implement effects on herbicide efficacy and the yield of cotton grown under a sprinkler or drip irrigation system. Weed Bio. Manage. 8(3): 201-208.

Paulsgrove, M.D., W.L. Barker, J.W. Wilcut. 2005. Bromoxynil-resistant cotton and selected weed response to mixtures of bromoxynil and pyrithiobac. Weed Tech. 19(3): 753761.

Prabhu, G., A.S. Halepyati, B.T. Pujari, B.K. Desai. 2011. Integrated weed management in Bt. cotton (GossypiumhirsutumL.) under irrigated conditions. Karnataka J. Agric. Sci. 24 (4): 529-530.

Reddy, N., Krishna, Locke, M. Martin, Koger, H. Clifford, Zablotowicz, M. Robert, L. J. Krutz. 2006. Cotton and corn rotation under reduced tillage management: impacts on soil properties, weed control, yield, and net return. Weed Sci.54 (4): 768774.

Riar, S. Dilpreet, Norsworthy, K. Jason, Griffith, M. Griff. 2011. Herbicide Programs for Enhanced GlyphosateResistant and Glufosinate-Resistant Cotton (Gossypiumhirsutum). Weed Tech. 25(4): 526-534.

Shaikh, M.A., A. Saleem and N.A. Malik. 2006. Integrated weed management and its effect on the seed cotton yield in cotton (Gossypiumhirsutum L.) croip. Pak. J. weed Sci. Res. 12 (1-2): 111-117. 